\title{
Special issue: Imaging of patients with pelvic floor disorders
}

\author{
Gaurav Khatri ${ }^{1} \cdot$ Kedar Jambhekar ${ }^{2}$ \\ Accepted: 16 March 2021 / Published online: 19 April 2021 \\ (c) The Author(s), under exclusive licence to Springer Science+Business Media, LLC, part of Springer Nature 2021
}

On behalf of the Pelvic Floor Disease Focused Panel (DFP), it gives us great pleasure to present this special issue on imaging of patients with pelvic floor disorders featuring scientific and review articles covering the different modalities used in pelvic floor imaging. This issue will serve as an update to a similar special pelvic floor imaging issue of Abdominal Imaging from 2013 and will both recapitulate and advance knowledge in several pertinent topics.

As is well known, the pelvic floor is a complex anatomic structure comprising of the pelvic floor muscles and various ligaments and fascia that work together as a cohesive unit to provide support for pelvic organs and function. Pelvic floor disorders comprise a myriad of abnormalities that are more prevalent than often appreciated and affect an increasingly aging population. With abnormalities that tend to simultaneously involve multiple compartments, comprehensive clinical evaluation can often be difficult resulting in incomplete patient evaluation. Dynamic imaging techniques can play a pivotal role in characterizing these multicompartmental abnormalities and thus augmenting clinical assessment by providing both anatomic and functional information. Fluoroscopy and magnetic resonance imaging (MRI) are the common modalities employed in patients with pelvic floor dysfunction. We also highlight the utility of pelvic floor ultrasound which is a powerful tool for certain pelvic floor indications. Although it has not been used as widely by radiologists in the past, it is employed more commonly in offices by pelvic floor surgeons. We hope to increase awareness and promote use of this modality among radiologists by including it in this issue.

Gaurav Khatri

Gaurav.Khatri@UTSouthwestern.edu

Kedar Jambhekar

KJambhekar@uams.edu

1 Department of Radiology, University of Texas Southwestern Medical Center, Dallas, TX, USA

2 Department of Radiology, University of Arkansas for Medical Sciences, Little Rock, AR, USA
A primary driving factor for the development and production of this special issue was to address the widespread inconsistencies in pelvic floor imaging technique and interpretation among radiologists. These inconsistencies are highlighted in this issue in a 'Perspectives' article that reports the results of an SAR membership survey on the use of pelvic floor imaging. In order to minimize such inconsistencies, many of the articles in this issue are authored by radiology experts from the Society of Abdominal Radiology (SAR) DFP on pelvic floor disorders including an SAR DFP consensus recommendations paper on MR defecography technique. Unique contributions in this issue include a multi-society opinion statement on clinical applications of the various pelvic floor imaging techniques endorsed by the SAR, American Urological Association (AUA) and American Urogynecologic Society (AUGS), as well as an article focused on practice improvement that provides tips on establishing and sustaining a successful pelvic floor MRI service. We also include a multi-modality review article on pelvic floor anatomy that sets the stage for subsequent modality specific articles on imaging findings in pelvic floor dysfunction. Dedicated articles on pelvic floor fluoroscopy, MRI, and ultrasound propose standardized techniques and appropriate reporting templates for each of the modalities. The issue also includes multiple original scientific papers related to MR defecography technique and correlation with clinical evaluation. Additional review articles discuss multi-modality imaging of functional defecatory disorders as well as postoperative imaging of synthetic materials used for pelvic floor repair.

We would like to thank all the contributors and authors who submitted their work for consideration in this special issue, including the members of the SAR Pelvic Floor DFP. We would also like to laud the efforts of our clinical colleagues and consultants for their invaluable insights and contributions to these works. We are honored to have served as the guest editors for this issue and wish to thank Dr. Daniel Johnson, the Editor-in-Chief, for this opportunity. This collaborative effort has certainly helped foster new relationships while procuring the expertise of both 
national and international experts on the subject matter. We are gratified by the work that is being done in the realm of pelvic floor imaging and hope that the readers will find this edition to be a valuable resource for their practice.

- Gaurav Khatri and Kedar Jambhekar
Publisher's Note Springer Nature remains neutral with regard to jurisdictional claims in published maps and institutional affiliations.

\section{(Guest Editors)}

